\title{
A mixed integer linear programming model for optimal sovereign debt issuance
}

\author{
P. Date ${ }^{\text {a }}$, A. Canepa ${ }^{\text {a }}$, M. Abdel-Jawad ${ }^{\text {a }}$ \\ ${ }^{a}$ Center for the Analysis of Risk and Optimization Modelling Applications, \\ Department of Mathematical Sciences, Brunel University, Middlesex UB8 3PH \\ $U K$
}

Keywords: multistage stochastic programming, public debt management

\begin{abstract}
Governments borrow funds to finance the excess of cash payments or interest payments over receipts, usually by issuing fixed income debt and index-linked debt. The goal of this work is to propose a stochastic optimization-based approach to determine the composition of the portfolio issued over a series of government auctions for the fixed income debt, to minimize the cost of servicing debt while controlling risk and maintaining market liquidity. We show that this debt issuance problem can be modeled as a mixed integer linear programming problem with a receding horizon. The stochastic model for the interest rates is calibrated using a Kalman filter and the future interest rates are represented using a recombining trinomial lattice for the purpose of scenario-based optimization. The use of a latent factor interest rate model and a recombining lattice provides us with a realistic, yet very tractable scenario generator and allows us to do a multi-stage stochastic optimization involving integer variables on an ordinary desktop in a matter of seconds. This, in turn, facilitates frequent re-calibration of the interest rate model and re-optimization of the issuance throughout the budgetary year allows us to respond to the changes in the interest rate environment. We successfully demonstrate the utility of our approach by out-of-sample back-testing on the UK debt issuance data.
\end{abstract}

\section{Introduction}

Governments borrow funds to finance the excess of cash payments over receipts, to pay interest on outstanding debt and to refinance maturing debt. The formulation of government debt strategies requires analyzing a complex dynamic inter-temporal problem as future costs and risks depend on many factors such as the size and structure of the existing debt and the evolution 
of the interest rates. When borrowing to finance the primary net funding requirement the government can choose from a number of different instruments. Examples include treasury bills, coupon bonds with fixed or inflation-linked coupons and retail saving bonds. The government wishes to select the composition and the maturity structure of its portfolio that minimize the cost of servicing the debt at a given risk. This task involves designing the maturity structure of the sovereign portfolio in such a way that the government's financing costs are kept low and insulated from macroeconomic shocks.

Most of the academic literature on optimal sovereign debt portfolio emphasizes the role of debt management in providing insurance against shocks as prescribed by optimal taxation theory with the final goal of stabilizing the debt-to-GDP ratio. According to this literature (see for example Barro (1997) or Missale (1999)), debt managers should minimize the risk that tax rates will have to be changed in response to economic developments. While offering many insights, this approach has few empirical implications. In practice, the majority of government debt managers make no explicit reference to fiscal policy, focusing instead on the budget-smoothing objective. Direct cost minimisation over a given fiscal horizon is rarely addressed.

In this article we focus on a specific goal, namely to determine the portfolio composition of the sovereign bond portfolio that minimizes the cost of servicing the debt while controlling risk and maintaining a certain level of market liquidity. We show that the problem of debt issuance through a series of auctions over time can be modeled as a stochastic optimization problem with a number of constraints imposed by the financial remit and government debt stabilization target.

The rest of the paper is organized as follows. Section 2 describes the debt management problem. Section 3.1 outlines the assumptions underlying the multistage stochastic optimization model. Section 3.2 describes notation used in setting up the MILP model for optimal debt issuance. Section 3.3 introduces a small (and less realistic) mixed integer linear programming (MILP) model, in order to get some insight into the modelling of the problem. Section 3.4 defines the risk measures considered here. Section 3.5 defines the actual stochastic optimization model. Sections 3.1-3.5 assume the knowledge of a recombining interest rate lattice which is derived separately. Sections 4.1-4.3 describe the filtering-based interest rate model, its calibration and its application in building a recombining interest rate lattice used in the earlier sections. The description of scenario generation model is separated from the description of the main optimization model in the interest of clarity. The effectiveness of the optimization model as well as scenario generation methodology is demonstrated through extensive out-of-sample numerical experiments using UK issuance data in section 5. Finally, section 6 summarizes the contributions of this paper and outlines the directions of future research. 


\section{The debt management problem}

Governments make use of debt instruments in order to finance two major components of the national accounts:

(1) the government net cash requirement, which is essentially the difference between government's income and expenditure in cash returns;

(2) the redemption of maturing government bonds. This is the amount needed to finance the annual repayment of maturing debt.

In managing the government debt, several governments have as their stated debt strategy objective the minimization of long-term financing cost while maintaining a low downside risk around those costs. In the UK, for example, the government explicitly states in HM Treasury (1998) that "the primary objective of debt management policy shall be to minimize, over the long term, the cost of meeting the Government's financing needs whilst:

- taking account of risk and

- so far as possible, to avoid conflict with monetary policy".

Although phrased in many different ways, similar statements relating the objective of government debt management are found in most of the Ministry of Finance code of practices around Europe and it is explicitly mentioned in the IMF guidelines for public debt management; see The International Monetary Fund and The World Bank (2003).

The trade-off between cost and risk is a familiar concept in the asset-pricing literature where investors attempt to optimally select the proportion of risky and riskless assets that maximize their expected utility functions subject to appropriate wealth constraints. This suggests that the government might be able to apply corporate finance theory in determining its debt issuance strategy. However, asset-liability management can not be applied to sovereign debt management in a straightforward manner. First, the objective and horizon of government debt management differ from those of private institutions and the types of risks actively managed at sovereign level also differ from private sector. In particular, while asset portfolio managers try to maximize asset returns over holding period subject to upper limit on risk, sovereign debt managers try to minimize the debt-service cost over a longer horizon subject to an implicit or explicit constraint on the volatility of debt-service cost(as a proxy for risk). Second, government debt managers are concerned with maintaining a liquid and well-functioning government security market. Sovereign fixed-income market often serve as a benchmark for corporate issuers, thus implying that small alterations of the government portfolio often have large impacts on the entire bond market. Therefore, the objective of minimizing the cost of debt servicing is subject to the constraint that a minimum level of bonds has to be issued at 
each maturity bracket. Finally, the implementation and transmission of monetary policy interventions occur through financial markets. According to the liquidity preference theory, debt management has a clear influence on the term structure of interest rates. Therefore, some constraints are imposed on debt management by the need to consider consistency with monetary policy.

The purpose of this paper is to integrate corporate portfolio optimization theory in a general framework which can be used by government debt managers to inform the issuance policy. In doing so we assume that one of the main sources of risk in sovereign debt portfolio management is the uncertainty about future short term interest rates. Other important sources of uncertainty such as the exposure to currency risk or fluctuations of macroeconomic variables (e.g., the rate of inflation) are not inserted directly into our cost minimization problem and are assumed to be closely correlated with the single source of uncertainty used. To model the evolution of interest rates, we use an affine term structure model introduced in Vasicek (1977) and calibrate it to multivariate time series data on government bond yields using a Kalman filter. This filtering-based calibration approach allows us to use the short term rate as an unobservable variable rather than using a proxy for it and to use potentially noisy yield data from which to estimate the short rate. Similar approaches have been previously employed in Babbs and Nowman (1999), Rossi (2004), Gravelle and Morley (2005) and Date and Wang (2009) among others. Date and Ponomareva (2010) provides a review of using Kalman filtering in financial time series models.

To generate scenarios of uncertain future interest rates (and hence the yields, which are affine functions of short rate for the chosen short term rate model) evolving through time, we use a trinomial recombining lattice. Using a recombining lattice is an industry standard way of modeling asset price or interest rate evolution for pricing purposes. In the present context, using a recombining lattice means that the number of possible values the yield vector can take grows linearly with time steps. In a non-recombining lattice, the number of steps can grow exponentially or combinatorially. The use of a recombining lattice keeps the mixed integer linear programming (MILP) based multi-stage stochastic programming problem numerically tractable, even on a desktop with modest hardware specifications. An alternative approach would be to use a non-recombining lattice followed by scenario generation heuristics, as proposed in Dupačová et al. (2003) and Heitsch and Römisch (2003).

The use of scenario based stochastic optimization in bond portfolio management is not new, although most of the applications are demand-side applications (i.e. the optimization problem as seen from the bond purchaser's point of view). A two stage stochastic program was formulated in Golub et al. (1995) to address fixed income portfolio management under interest rate and cashflow uncertainty, while a similar formulation was used in Zenios (1995) to illustrate 
management of portfolios containing mortgage backed securities. In Dupačová and Bertocchi (2001), bond portfolio management is formulated as a multiperiod stochastic program in a dynamic setting. Like our paper, Dupačová and Bertocchi (2001) also uses a recombining lattice as a temporal model for uncertain interest rates.

On the supply side (i.e., for a sovereign issuance problem), a linear programming based model is presented in Adamo et al. (2004) for minimization of the total cost of issuance under regulatory constraints. This model is illustrated using debt issuance data of the Italian government. Other notable work in this area includes Bernaschi et al. (2007), which provides results on the multivariate simulation of interest rates using observable (ECB) rates as well as analysis of principal components. The research reported in Consiglio and Staino (2010) and Balibek and Köksalan (2010) is the closest in spirit to the work reported here, in the sense that, both these papers also develop multi-stage stochastic programming models for sovereign debt issuance.

Our model differs from the earlier work mentioned above in several aspects. These are summarized as follows.

(1) We calibrate the interest rate model using a Kalman filter and noisy yield measurements and use this to create bond price scenarios for the optimization model. Arguably, this reflects better market expectation of the bond prices obtainable through auctions than using primary economic variables. Using a filter based interest rate model also allows for easy recalibration and hence allows for generating interest rate scenarios which are tuned to more recent market data. For demand-side optimization, a similar approach was taken in Nielsen and Poulsen (2004) where a two factor interest rate model is used along with a multi-factor stochastic program to manage mortgage-backed securities. Filtering-based model is also used in a simulation framework in the report by Danish National Bank (2006). The authors are not aware of the use of filtering based framework to generate scenarios in a supply-side optimization.

(2) We use a recombining lattice-based stochastic programming model as opposed to a non-recombining scenario tree used in Consiglio and Staino (2010) and Balibek and Köksalan (2010) while discussing the sovereign debt issuance. This makes the problem computationally significantly simpler, as the number of scenarios is reduced significantly, while retaining consistency with the underlying theoretical interest rate model.

(3) We use a receding horizon approach to carry out multiple, multistage stochastic programs over a period of time to optimize debt issuance cost over a given horizon; see Maciejowski (2002) for control engineering applications of the receding horizon approach. Once a stochastic programming exercise is carried out, one need not stick to the full sequence of optimal decisions with passage of time, as the uncertainty progressively 
resolves itself. We propose re-calibrating the scenario generation (i.e. interest rate ) model periodically and use it to re-optimize the issuance over the remaining period, using the issuance data up to that time. To our knowledge, the use of receding horizon strategy in an optimization model is new.

(4) Finally, we carry out out-of-sample back-testing to compare the performance of our strategy against the actual debt issuance by the UK government in the budgetary years 2006-2008. Our results show that a significant debt-service cost reduction can be achieved by carrying out a rigorous optimization exercise. Note that the interest rate model used for scenario generation is calibrated on one data set and the optimization is carried out on a different (out-of-sample) data set throughout this exercise and the actual issuance decisions are not used as inputs to the model.

\section{The multistage stochastic optimization model}

\subsection{Assumptions}

We start with the following assumptions about the process of raising debt by a sovereign government.

(1) The sovereign body raises debt through a series of auctions. At the beginning of the financial year, the dates of debt auctions are fixed. There are three separate auction calenders; one each for short, medium and long dated bonds. Imperatives other than purely financial ones play a significant role in deciding this calendar and we consider these calendars as input data.

(2) At each auction, a single bond is issued, either from the existing (or pre-issued) stock or a bond with new maturity.

(3) The average price of any bond at the auction is its arbitrage free price as determined by the yield curve. The yield of any new bond issued is also determined by the yield curve on the auction date.

(4) Fiscal policy is responsible for the government net cash requirement. Thus, the total amount to be raised over the financial year is dictated by the government's borrowing requirements and is assumed to be an exogenous constant. Further, the total amounts to be raised through each set of auctions viz. auctions of short, medium, long dated bonds, are fixed. These are dictated by the government's need to maintain liquidity in markets of bonds with different maturities.

(5) The government does not engage in opportunistic borrowing. As a consequence financial strategies that attempt to take advantage of the market conditions for issuance of various debt instruments are ruled out. This op- 
erational principle together with the need of pursuing an issuance policy that is open and transparent are often described in the code of practice of the debt managing agencies.

Under these assumptions, the optimization model uses a receding horizon approach as mentioned earlier; see e.g. Maciejowski (2002) for applications in control engineering. This approach in the present context may be explained as follows. Suppose that there are $N$ auction dates indexed from $t_{1}$ through $t_{N}$. At each auction date $t_{i}, i>1$, data of previous auctions $t_{1}, \cdots t_{i-1}$ are already available. Also, the multivariate time series data are available until time $t_{i}$. It is assumed that, at each $t_{i}$, a new recombining interest rate lattice is established and a multi-state stochastic optimization problem is solved to generate the choice of bond and the amount of debt to be auctioned from $t_{i}$ onwards, i.e. at $t_{i}, t_{i+1}, \cdots, t_{N}$. If $\mathcal{T}_{i}$ is the number of stages in the stochastic optimization problem solved at $t_{i}$, then $\mathcal{T}_{i}-\mathcal{T}_{j}=i-j$ for $1 \leq i \leq j \leq N$. In the numerical experiments reported in 5 , this receding horizon approach is followed based on real UK data and the results are compared with the actual issuance during the same period.

\subsection{Notation}

As outlined later in section 4.3, we use a re-combining tree (or lattice) to model the evolution of interest rates. For a re-combining tree, the number of nodes (and hence decision variables) grows linearly with time and the problem remains tractable even for a long time horizon. Given an interest rate lattice and hence a set of scenarios for future bond yields, we outline here the notation used in our development of the optimization model. The scenario generation will be discussed separately in latter subsections.

(1) $\mathcal{N}=\{1,2, \cdots, N\}, \mathcal{J}=\{1,2, \cdots, J\}$ and $\mathcal{K}=\{1,2, \cdots, K\}$ are the index sets for auctions over the budget year, interest rate scenarios and bonds to be auctioned respectively.

(2) $X_{i . j}^{(k)}$ is a binary decision variable which has a value 1 if $k^{\text {th }}$ gilt is auctioned at $i^{\text {th }}$ auction, in $j^{\text {th }}$ scenario; $X_{i j}^{(k)}$ is 0 otherwise.

(3) $u_{i . j}^{(k)}$ is a real valued decision variable which gives the number of units of bond $k$ sold at auction $i$ in $j^{\text {th }}$ scenario. The unit is enforced to be a multiple of a constant $\gamma$ in the model with the use of $\omega_{i . j}$ as an integer variable.

(4) $P_{i . j}^{(k)}$ is the forecasted price of the $k^{t h}$ bond at the $i^{\text {th }}$ auction in the $j^{\text {th }}$ scenario; this is explained in more detail later in section 4.3.

(5) The amount raised at a single auction is bounded from above by $\bar{D}$ and from below by $\underline{D}$, both of which are specified constants.

(6) $D 0_{i . j}^{(k)}$ is the prior holding of the $k^{\text {th }}$ bond at the $i^{\text {th }}$ auction in the $j^{\text {th }}$ sce- 
nario. $\bar{\psi}$ is the upper bound for the liquidity constraint this is a constant for a specific problem to make sure that to many already existing bonds don't exist.

(7) $\tau_{i}^{(k)}$ is time to maturity of bond $k$ starting from time $t_{i}$. It needs to satisfy the maturity constraint, i.e. $\underline{\tau} \leq \tau_{i}^{(k)} \leq \bar{\tau}$, where $\underline{\tau}, \bar{\tau}$ are given constants.

(8) $B \geq 1$ is a constant integer which limits the number of times a specific bond can be used in the considered financial year. The choice of integer $B$ is a trade-off between flexibility in choosing the lowest cost issuance and ensuring enough liquidity across all maturities.

(9) $L$ is the principal of each bond. $I_{j}$ represents the total cost of issuance over the lifetime of debt in scenario $j$ :

$$
I_{j}=\sum_{i \in \mathcal{N}} \sum_{k \in \mathcal{K}} u_{i . j}^{(k)} L\left(1+C_{i}^{(k)}\right)
$$

where $C_{i}^{(k)}$ represents the total amount of coupons over the remaining life of bond $k$ from time $t_{i}$ onwards. This cost function is calculated in accordance with the European System of Accounts (ESA95).

\subsection{A simplified optimization model for the debt issuance problem}

For the set-up outlined above, it is worth considering a deterministic optimization problem first. Let us assume that future prices are "known" as $P_{i}^{(k)}$ for the auction date at $t_{i}$ and for the unit of bond $k$, the auctions will sell out. Thus the second subscript for prices $P$, which indexes the scenarios, is not used and the overall notation is simplified.

Let $X_{i}^{(k)}$ be the binary variable that represents which bond $k$ to issue at the $i^{\text {th }}$ auction and $u_{i}^{(k)}$ be a real variable that estimates the amount of bonds to issue for $k$ bond at $i^{t h}$ auction date. The total cost is simply the un-discounted total cash flow from the issuance of the year. The simplified version of optimization model without uncertainty can then be expressed as follows.

$$
\begin{aligned}
& \text { minimize } \sum_{i \in \mathcal{N}} \sum_{k \in \mathcal{K}} u_{i}^{(k)} L\left(1+C_{i}^{(k)}\right) \text { subject to } \\
& \sum_{(i, k) \in(\mathcal{N}, \mathcal{K})} u_{i}^{(k)} P_{i}^{(k)} \geq D, \\
& u_{i}^{(k)} P_{i}^{(k)}+D 0_{i}^{(k)} \leq \bar{\psi}-\sum_{p t=1}^{i} u_{p t}^{(k)} P_{p t}^{(k)} \forall i \in \mathcal{N}, k \in \mathcal{K},
\end{aligned}
$$




$$
\begin{aligned}
& \underline{D} X_{i}^{(k)} \leq u_{i}^{(k)} P_{i}^{(k)} \leq \bar{D} X_{i}^{(k)} \forall i \in \mathcal{N}, k \in \mathcal{K} \\
& \sum_{(i, k) \in(\mathcal{N}, \mathcal{K})} X_{i}^{(k)}=N \\
& \sum_{k \in \mathcal{K}} X_{i}^{(k)}=1, \forall i \in \mathcal{N} \\
& \sum_{i \in \mathcal{N}} X_{i}^{(k)} \leq B, \forall k \in \mathcal{K}
\end{aligned}
$$

The equations in this model can be explained as follows.

- Inequality (3.2) guarantees that the minimum required amount of debt is raised over through the specified series of auctions.

- Inequality (3.3) ensures that the total issuance for a particular bond (or a particular maturity) remains under a specified constant $\bar{\psi}$.

- Inequality (3.4) constrains the minimum and the maximum issuance size at each auction.

- Equations (3.5)-(3.6) ensure that all auctions are used and only one bond is issued at each auction.

- Finally, (3.7) is a constraint to ensure that one bond is used at most $B$ times in the series of auctions.

Analytically, this model can be solved using a deterministic mixed integer linear program, with the amounts auctioned and the issuance choice (binary) variables as the decision variables. Although the model constitutes a useful exercise, it is overly simplified to illustrate the issues involved in public debt issuance. The assumption that prices are known and a lack of measure to control the issuance risk make the problem highly unrealistic. In the subsequent sections, we will introduce the necessary risk measures and will also introduce a mechanism to generate scenarios for different possible future prices for bonds.

\subsection{Risk Measures}

Risk measures provide information about the uncertainty of future debt-service cost, therefore the value at risk plays a central role in the management of government debt. An increase in the value of the debt portfolio reflects an increase in the future burden for taxpayers or it may boost the cost of other debt instruments often used by debt managers such as swaps or buybacks.

As a measure of risk, we use two different measures: Conditional Value at Risk (CVaR) and a quantile based supply-side measure called Cost at Risk (CaR), as discussed in Risbjerg and Holmund (2005).

The CVaR risk measure is used as a system of linear constraints as defined 
in Rockafellar and Uryasev (2000). The CVaR constraint is also bounded to control the maximum amount of conditional risk tolerated. A similar bound is explained in Consiglio and Staino (2010):

$$
C V a R:=\frac{\sum_{j \in \mathcal{J}} p_{j} \phi_{j}}{1-\beta}+\zeta
$$

with

$$
\phi_{j}:=\max \left(I_{j}-\frac{\sum_{j \in \mathcal{J}} I_{j}}{J}-\zeta, 0\right),
$$

where $\mathcal{J}$ is an index set as defined in section $3.2, \zeta \in \mathbb{R}, p_{j}$ is the probability of the $j^{\text {th }}$ scenario, or a branch of the tree to occur and $\beta$ corresponds to the confidence rate between 0 and 1 . In the proposed model, the value of CVaR will be bounded from above by a constant $\rho$. We also consider that each branch will have an equal probability to occur, so that we can take $p_{j}$ to be $\frac{1}{J}$. Now the CVaR constraint becomes:

$$
C V a R:=\frac{\sum_{j \in \mathcal{J}} \phi_{j}}{J(1-\beta)}+\zeta
$$

As the CVaR upper bound is reduced, the difference between costs of different scenarios is reduced as well. As theory suggests, this will raise the expected cost in general.

The CaR measure is defined in Hahm and Kim (2003) as:

$$
C a R:=\mathbb{E}\left(I_{j}\right)+1.645 \varsigma,
$$

where $\mathbb{E}\left(I_{j}\right)=\sum_{j \in \mathcal{J}} I_{j} / J$ and $\varsigma$ is the standard deviation of the achieved cost. This supply side measure is similar to the popular Value at Risk (VaR) measure on the demand side, under the assumption of normally distributed scenarios. In our case, the standard deviation is computed a posteriori as the sample standard deviation over all the scenarios.

\subsection{Definition of the optimization model}

The mixed integer linear programming model for the optimal debt issuance problem is defined as follows.

$$
\operatorname{minimize} \frac{1}{J} \sum_{j \in \mathcal{J}} I_{j} \text { subject to }
$$




$$
\begin{aligned}
& \sum_{(i, k) \in(\mathcal{N}, \mathcal{K})} u_{i . j}^{(k)} P_{i . j}^{(k)} \geq D, \forall j \in \mathcal{J}, \\
& u_{i . j}^{(k)} P_{i . j}^{(k)}=\gamma \omega_{i . j}, \forall i \in \mathcal{N}, k \in \mathcal{K}, j \in \mathcal{J} \\
& \phi_{j}=I_{j}-\frac{1}{J} \sum_{j \in \mathcal{J}} I_{j}-\zeta, \forall j \in \mathcal{J}, \\
& \frac{1}{J(1-\beta)} \sum_{j \in \mathcal{J}} \phi_{j}+\zeta \leq \rho \\
& u_{i . j}^{(k)} P_{i . j}^{(k)}+D 0_{i . j}^{(k)} \leq \bar{\psi}-\sum_{p t=1}^{i} u_{p t . j}^{(k)} P_{p t . j}^{(k)} \forall i \in \mathcal{N}, k \in \mathcal{K}, j \in \mathcal{J}, \\
& \underline{D} X_{i . j}^{(k)} \leq u_{i . j}^{(k)} P_{i . j}^{(k)} \leq \bar{D} X_{i . j}^{(k)} \forall i \in \mathcal{N}, k \in \mathcal{K}, j \in \mathcal{J}, \\
& \sum_{(i, k) \in(\mathcal{N}, \mathcal{K})} X_{i . j}^{(k)}=N, \forall j \in \mathcal{J}, \\
& \sum_{k \in \mathcal{K}} X_{i . j}^{(k)}=1, \forall i \in \mathcal{N}, j \in \mathcal{J}, \\
& \sum_{i \in \mathcal{N}} X_{i . j}^{(k)} \leq B, \forall j \in \mathcal{J}, k \in \mathcal{K}, \\
& i f \underline{\tau} \geq \tau_{i}^{k} \text { or } \bar{\tau} \leq \tau_{i}^{k} t h e n X_{i . j}^{k}=0 \forall i \in \mathcal{N}, j \in \mathcal{J} .
\end{aligned}
$$

The optimization procedure is schematically illustrated in figure 1. The goal

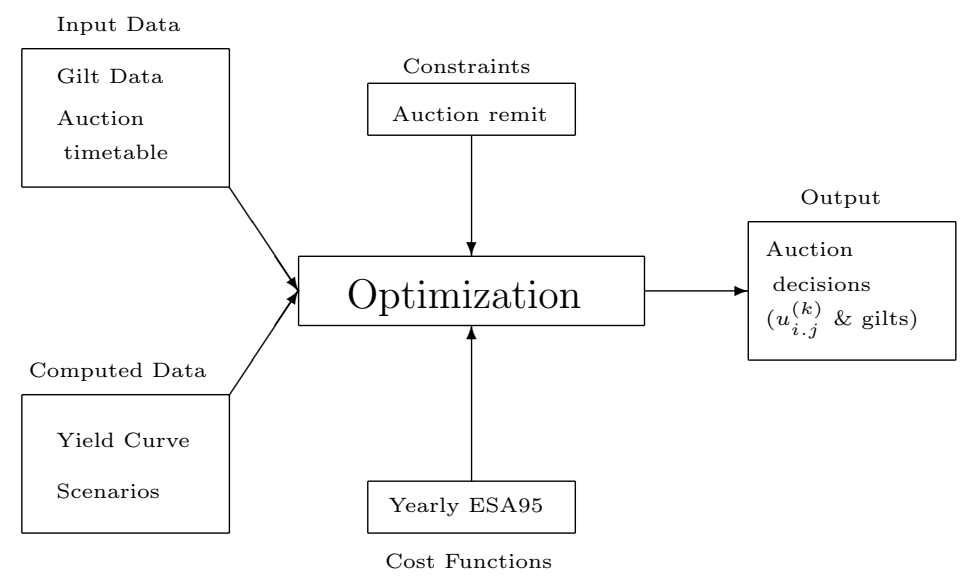

Fig. 1. Optimization procedure 
is to minimize the average cost of debt servicing as defined by ESA95 over all interest rate scenarios $\mathcal{J}$ and the set of auctions $\mathcal{N}$. The rest of the notation in the above model is as defined in section 3.2 and the model is subject to assumptions stated in section 3.1. The set of equations is an expanded version of the model presented earlier in section 3.3 and can be explained as follows.

- Inequality (3.9) is a constraint to make sure the amount raised is at least the fixed objective $D$ over the year.

- Equation (3.10) exists to ensure that the auctions are done in increments of $\gamma, \omega_{i . j}$ being an integer variable to ensure the increments are respected.

- The systems of inequalities (3.11)-(3.12) corresponds to the CVaR risk measure bounded from above by $\rho$ with confidence $\beta$.

- Equation (3.13) is a liquidity constraint and ensures that the total amount of a specific bond in issuance doesn't exceed an upper bound $\bar{\psi}$.

- Equation (3.14) ensures that each auction will raise funds within the boundaries set by a government.

- Equations ( 3.15)-(3.16) impose constraints that all auctions are used and only one bond is auctioned on each auction date.

- The inequality (3.17) ensures that a single bond is used no more than $B$ times.

- Finally, the last constraint (3.18) ensures that if the maturity of a particular bond does not match the maturity constraint of a problem at the $i^{\text {th }}$ auction it may not be auctioned by the model.

The optimization model discussed so far assumes that a mechanism is available for generating scenarios of bond prices. These scenarios need to be arbitragefree, since we are assuming that the auction prices are determined by the secondary market. In the next section, we discuss the stochastic interest rate model for generating these price scenarios (or equivalently, bond yield scenarios) and also outline our method of calibrating this model.

\section{Generation of bond price scenarios for optimization}

\subsection{Vasicek term structure model}

Future debt costs and future debt financing are subject to unknown future interest rates. In order to assess and quantify the interest rate costs it is necessary to model the future evolution of interest rates at which the future debt financing takes place. The uncertainty about future interest rates needs to be modelled in a fashion which is both realistic and tractable. For this purpose, we assume that the instantaneously compounded interest rate (or short rate), $r_{t}$, follows the affine Gaussian dynamics first proposed in Vasicek 
(1977):

$$
d r_{t}=a\left(b-r_{t}\right) d t+\sigma d W_{t},
$$

where $a, b$ are constants and $\sigma$ is the volatility considered constant here as well. $W_{t}$ is the standard Brownian motion. The mean and the variance of $r_{t+\Delta}$ conditional on $r_{t}, \Delta \geq 0$, is given by:

$$
\begin{aligned}
\mathbb{E}\left(r_{t+\Delta} \mid r_{t}\right) & =\bar{b}+e^{-a \Delta}\left(r_{t}-\bar{b}\right), \\
\operatorname{Var}\left(r_{t+\Delta} \mid r_{t}\right) & =\frac{\sigma^{2}}{2 a}\left(1-e^{-2 a \Delta}\right) .
\end{aligned}
$$

where $\bar{b}=b-\frac{\lambda \sigma}{a}$ and $\lambda$ is the price of risk which is assumed to be constant through time.

The price of a zero-coupon bond maturing at time $T(T>t)$, at time $t$ can be proved to be:

$$
P(t, T)=A(t, T) e^{-B(t, T) r(t)},
$$

where $A, B$ are functions of time to maturity $T-t$ and are given by

$$
\begin{aligned}
& B(t, T)=\frac{1}{a}\left[1-e^{-a(T-t)}\right], \\
& A(t, T)=\exp \left\{\left(\bar{b}-\frac{\sigma^{2}}{2 a^{2}}\right)[B(t, T)-\Delta]-\frac{\sigma^{2}}{4 a} B(t, T)^{2}\right\} .
\end{aligned}
$$

where a, $\sigma$ are defined in 4.1. Finally, the yield (return on the bond) corresponding to a zero coupon bond at time $t$, maturing at a time $T>t$, is given by:

$$
y(t, T)=-\frac{1}{T-t} P(t, T)=\log (A(t, T))-B(t, T) r_{t} .
$$

Remark: Unlike most of the conventional literature on simulation of debt issuance (see, e.g. Bernaschi et al. (2007)), we do not use macroeconomic variables to model the evolution of interest rates. Our supporting argument is that, the secondary sovereign debt market for OECD countries is quite liquid and reflects the view of the market participants about the evolution of macroeconomic variables. It makes sense to model the interest rate movement based on the data which reflects the "demand-side" view of these participants when one is making decisions about auctioning debt. Alternative models are possible; see e.g. James and Webber (2000) for a variety of short rate models and Diebold and Li (2006) for a dynamic version of the commonly used Nelson-Siegel model first proposed in Nelson and Siegel (1987). Our choice of a one factor Gaussian model allows us to use a one dimensional recombining lattice to represent the uncertainty in the interest rates in a realistic, yet computationally tractable fashion, as explained later in Section 4.3. 


\subsection{Kalman filtering-based calibration}

To set up a calibration problem formally in discrete time, we need to discretise (4.1). From (4.2)-(4.3), a natural discretisation of (4.1) which preserves the conditional mean and the variance of $r_{k+1}$ at time $t_{k+1}$ is given by

$$
r_{k+1}=\bar{b}+e^{-a \Delta t}\left(r_{k}-\bar{b}\right)+\sqrt{\frac{\sigma^{2}}{2 a}\left(1-e^{-2 a \Delta t}\right)} \epsilon_{k+1}
$$

where $\left\{\epsilon_{k}\right\}$ is a sequence of scalar i.i.d. Gaussian random variables with zero mean and unit variance and $\Delta=t_{k+1}-t_{k}$ is assumed to be a constant for all $k \cdot r_{k}$ is an unobserved variable as there is no observable security which pays return instantaneously. Instead, one may observe yields $y\left(t_{k}, T_{i}\right)$ as defined in (4.7) from zero coupon bonds at each time $t_{k}$, for different maturities $T_{1}, T_{2}, \ldots T_{N}, T_{i}>t_{k}$. To describe the observation equation, denote by $\mathbf{y}_{k}$ a vector in $\mathbb{R}^{N}$ whose $i^{\text {th }}$ element is $y\left(t_{k}, T_{i}\right)$. Time dependent vectors $\mathbf{A}_{k}$ and $\mathbf{B}_{k}$ are defined similarly using (4.5)-(4.6). Then one can express the yield vector as an affine function of short rate $r_{k}$ as

$$
\mathbf{y}_{k}=\log \left(\mathbf{A}_{k}\right)-\mathbf{B}_{k} r_{k} .
$$

Further, one may assume that our model of the short rate is imperfect and that the vector of observed yields at time $t_{k}$ is given by

$$
\mathbf{R}_{k}=\mathbf{y}_{k}+\sigma_{y} \mathbf{e}_{k}
$$

where $\left\{\mathbf{e}_{k}\right\}$ is a vector valued, i.i.d. Gaussian sequence with zero mean and identity matrix as covariance and $\sigma_{y}>0$ is a constant indicating the dispersion of the observed yields from their value given by the model. (4.8) and (4.10) form a linear state space system. We can use Kalman filter to calibrate the model from observed time series $\mathbf{r}_{k}, k=1,2, \ldots, M$. The recursive equations for the Kalman filter are repeated below for easy reference. The optimal estimate of $r_{k+1}$ based on measurement $\mathbf{R}_{k}$ (respectively, based on $\mathbf{R}_{k+1}$ ) is denoted as $\hat{r}_{k+1 \mid k}$ (respectively, $\left.\hat{r}_{k+1 \mid k+1}\right)$. $\mathbf{v}_{k}$ denotes the innovations vector at time $t_{k}$ while $\Sigma_{k}$ denotes the covariance matrix of innovations at time $t_{k}$. The set of equations given below outline the recursive propagation of estimates from $\hat{r}_{k \mid k-1}, P_{k \mid k-1}$ to $\hat{r}_{k+1 \mid k}, P_{k+1 \mid k}$ after measuring $\mathbf{R}_{k}$.

$$
\begin{aligned}
\mathbf{v}_{k} & =\mathbf{R}_{k}-\log \left(\mathbf{A}_{k}\right)-\mathbf{B}_{k} \hat{r}_{k \mid k-1}, \\
\Sigma_{k} & =\sigma_{y}^{2} I+\mathbf{B}_{k} P_{k \mid k-1} \mathbf{B}_{k}^{\top}, \\
\hat{r}_{k \mid k} & =\hat{r}_{k \mid k-1}+P_{k \mid k-1} \mathbf{B}_{k} \Sigma_{k}^{-1} \mathbf{v}_{k}, \\
\hat{r}_{k+1 \mid k} & =\bar{b}+e^{-a \Delta}\left(\hat{r}_{k \mid k}-\bar{b}\right), \\
P_{k+1 \mid k} & =e^{-2 a \Delta} P_{k \mid k-1}+\frac{\sigma^{2}}{2 a}\left(1-e^{-2 a \Delta}\right)-e^{-2 a \Delta} P_{k \mid k-1}^{2} \mathbf{B}_{k}^{\top} \Sigma_{k}^{-1} \mathbf{B}_{k} .
\end{aligned}
$$


The joint probability density function (also called the likelihood function) of

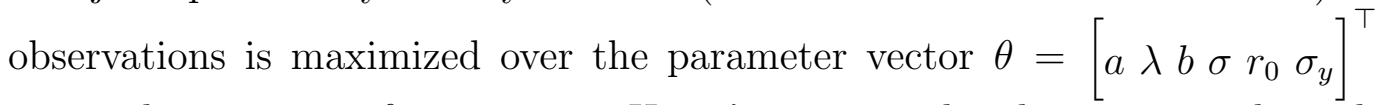
to get the estimate of parameters. Here $\lambda$ is assumed to be constant through time. Since the forecast errors are i.i.d., the log likelihood function is expressed by:

$$
L(\theta)=\sum_{k=1}^{M} \log p\left(\mathbf{R}_{k} \mid \mathcal{F}_{k-1}, \theta\right) .
$$

Further, as the errors are Gaussian, maximizing the likelihood function $L$ is also equivalent to minimizing

$$
-L\left(\mathbf{R}_{k}, \theta\right)=\frac{1}{2} \sum_{k=1}^{M}\left(\log \operatorname{det}\left(\Sigma_{k}\right)+\mathbf{v}_{k}^{T} \Sigma_{k}^{-1} \mathbf{v}_{k}\right),
$$

where the constant terms are ignored. This smooth nonlinear cost function can be minimized over the set of parameters using any standard nonlinear solver. We use MATLAB's "off-the-shelf" optimizer fminsearch which seemed to perform satisfactorily.

\subsection{Re-combining lattice for interest rates}

As in the previous section, after each (re-)calibration we build a re-combining trinomial lattice using a procedure in Hull and White (1994) and use it for setting up an optimization problem at each auction. This idea of solving multiple, possibly multi-stage optimization problems during the financial year is realistic as the sovereign debt issuing authority can dynamically adjust its decisions during the year as the economic environment evolves. This idea of using multiple optimizations over the trajectory of an uncertain variable is similar to the receding horizon approach used in predictive process control, see e.g. Maciejowski (2002). We build a $Q$ step lattice at the beginning of each quarter using the parameters of recent calibration. A construction for $Q=3$ is shown in figures 2-3 to explain the idea of a receding horizon. We take the short rate at each node to be:

$$
r_{i}^{(j)}= \begin{cases}r_{i-1}^{(j)} \exp \left(\frac{2 \sigma^{2}}{a}\left(1-e^{-a \Delta}\right)\right) & \text { for the upper branch of the lattice } \\ r_{i-1}^{(j)} & \text { for the middle branch of the lattice } \\ r_{i-1}^{(j)} \exp \left(-\frac{2 \sigma^{2}}{a}\left(1-e^{-a \Delta}\right)\right) & \text { for the lower branch of the lattice. }\end{cases}
$$

The forecasted short rate used for future auctions is then linearly interpolated from the tree if the auction date does not coincide with a tree node. Using the Vasicek pricing formula, we can obtain the price of a bond with maturity $T_{k}$, 
at time $t_{i}$ and corresponding to a short rate $r_{i}^{(j)}$ by summing over all coupons:

$$
P_{i . j}^{(k)}=\sum_{t_{i}<t_{c} \leq T_{k}} c^{(k)} A\left(t_{i}, t_{c}\right) e^{-B\left(t_{i}, t_{c}\right)} r_{i}^{(j)}+L A\left(t_{i}, T_{k}\right) e^{-B\left(t_{i}, T_{k}\right)} r_{i}^{(j)}
$$

where $L$ is the principal of each bond, $c^{(k)}$ is the coupon of the $k^{t h}$ bond, $t_{c}$ belongs to the the set of maturities of all the remaining coupons for the bond considered and $A(t, T), B(t, T)$ are as defined in section 4.1.

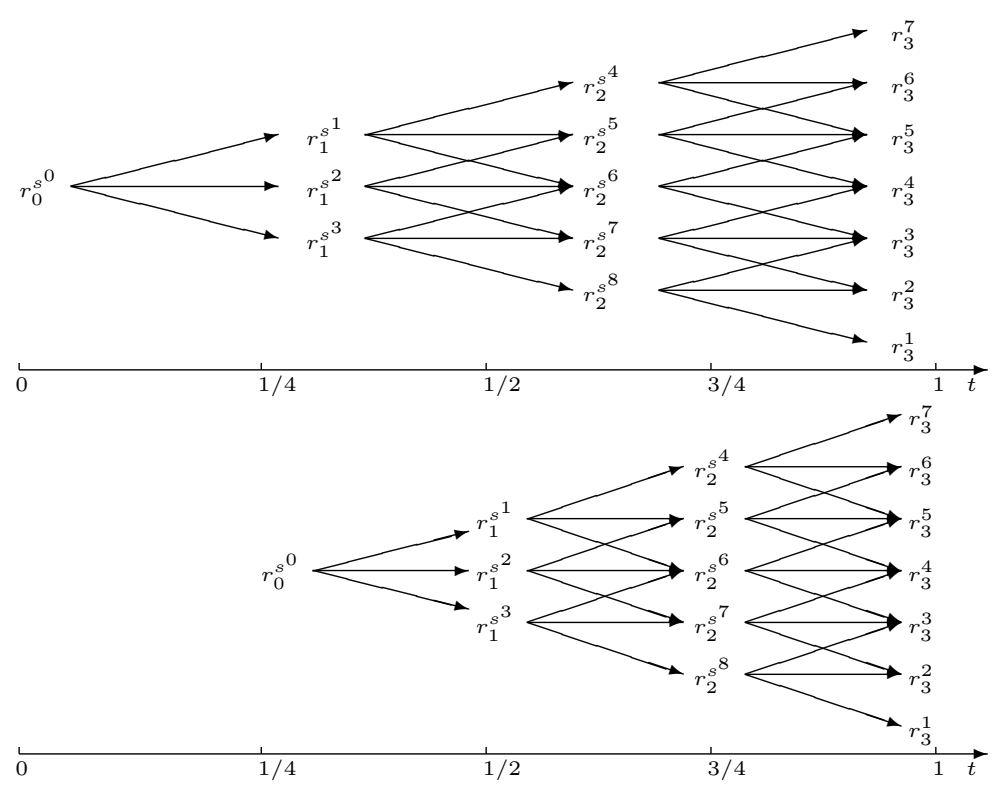

Fig. 2. Lattice at the beginning of the $1^{\text {st }}$ and $2^{\text {nd }}$ quarter.

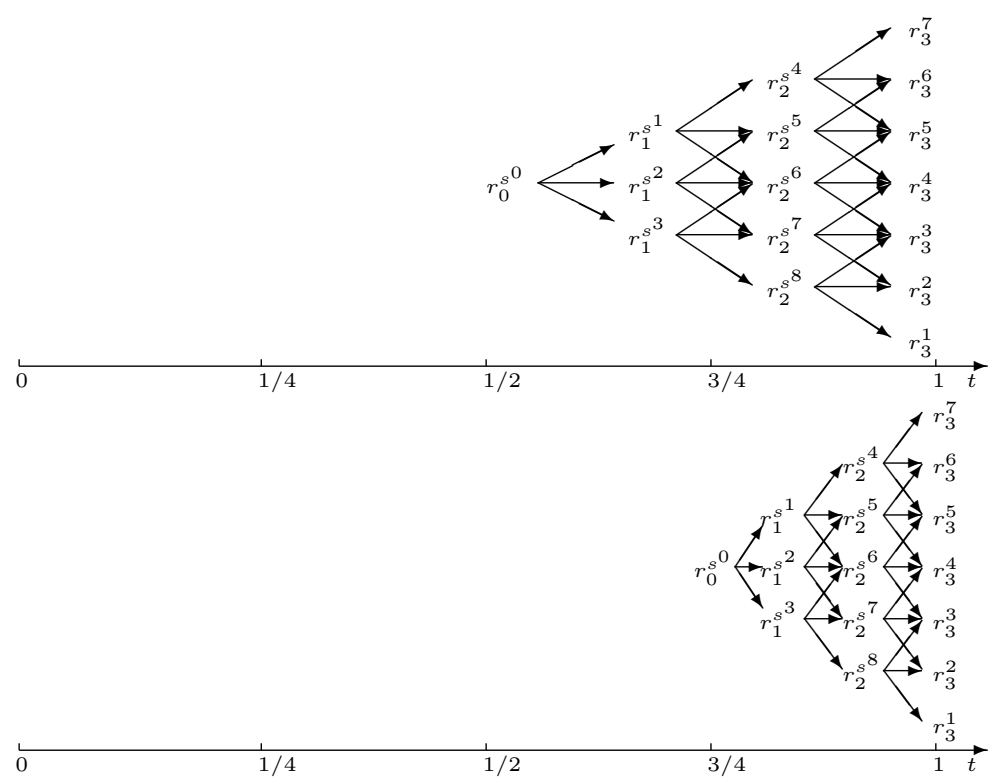

Fig. 3. Lattice at the beginning of the $3^{\text {rd }}$ annd $4^{\text {th }}$ quarter. 


\section{An Application to the UK Government Debt Portfolio}

\subsection{Problem parameters}

We apply the proposed optimization model to the UK government debt problem for the $2007-08$ year. The model parameters $N, K$ and $D$ for the debt problem (auctions, bonds and amounts to be raised), as defined in section 3.2 are:

\begin{tabular}{|l|c|c|c|}
\hline Subproblem & N & K & D (in bn) \\
\hline short $(1-7$ years $)$ & 4 & 16 & 10 \\
medium $(7-15$ years $)$ & 4 & 8 & 10 \\
long $(>15$ years $)$ & 11 & 10 & 23.4 \\
\hline
\end{tabular}

Table 1. Parameters used for optimization.

As well as using the real bonds that were available during that financial year, some of the parameters of the optimization models are chosen based on the government remit as follows with those defined in table 1.

- $\gamma=250$ is the amount in million pound sterling to increment the amount raised at an auction.

- $\underline{D}=1,500$ million and $\bar{D}=4,000$ million, these are set in the remit.

- $B=2$ is the maximum amount of times we choose to issue a particular bond in the set of auction considered for short dated and medium dated bonds. $B=3$ for the long dated bonds issuance problem.

We will refer to the problem of issuance of short dated bonds as the short subproblem. Similarly the medium dated bonds and long dated bonds correspond to the medium subproblem and the long subproblem respectively. The CVaR measure of risk will be compared to the traditional VaR measure and the CaR measure introduced in Hahm and Kim (2003). $\bar{\psi}$ is 20 billion for the short subproblem, 22 billion for the medium subproblem and 40 billion for the long subproblem. The use of longer term debt doesn't decrease the expected cost, as the cost function takes into account all coupon and principal repayments, not discounted by the effect of inflation. The effect of inflation reduces quite considerably the actual cost of the debt. 


\subsection{Calibration of interest rate model}

We use the procedure in section 4.2 to calibrate a one factor Gaussian term structure model to UK government bond data. Gilt yields from 2006 to 2008, obtained from the UK Debt Management Office, were used for the numerical experiments. We use daily data from April 2006 to March 2008. The model is calibrated every quarter from March 2007 to March 2008, based on the past data stretching back one year. In other words, we move the one year calibration window forward through time as the year unfolds into the next financial year. The re-calibration takes into account the fact that the interest rate model parameters may not be constant and drift through time, e.g. due to the impact of the earlier issuance and due to the changes in the market sentiment. The choice of re-calibration every quarter corresponds to quarterly review. We take 26 long, short and medium dated bonds over that period and calibrate over 506 yields per bond, i.e. a total of 13,156 yields were used. The parameters obtained through four calibration experiments are listed in table 1 below. It can be seen that only the long run mean $b$ shows a significant variation through time. We describe how these parameters are used to construct a trinomial tree at each quarter for scenario based optimization in the next section.

\begin{tabular}{|l|c|c|c|c|c|c|}
\hline time & $a$ & $\lambda$ & $b$ & $\sigma$ & $r$ & $\sigma_{y}$ \\
\hline 0 months & 0.114278 & 0.070403 & 0.060242 & 0.036074 & 0.067142 & 0.010748 \\
3 months & 0.114278 & 0.070403 & 0.063255 & 0.036074 & 0.067142 & 0.010748 \\
6 months & 0.114278 & 0.070403 & 0.066417 & 0.036074 & 0.067142 & 0.010748 \\
9 months & 0.114278 & 0.070403 & 0.069738 & 0.036074 & 0.067142 & 0.010748 \\
\hline
\end{tabular}

Table 2. Parameters of Vasicek model (UK 2006-2008 data)

\subsection{Numerical results for the optimization model}

A sequence of mixed integer linear programming problems, with problem data as in section 5.1 and scenarios generated using parameters in section 5.2, are solved using CPLEX version 11.0.0 running on Intel dual core, $2.66 \mathrm{GHz}$ processor with 3.24GB RAM. Due to relatively small number of integer variables (with at most 16 bonds in any one subproblem), the problem can be solved quite quickly even for multiple stages. In tables 3-6, the solving time (S.T.) is in seconds, while the standard deviation and the risk measures are in million pound sterling. $Q$ is the number of stages for stochastic program solved after each (re-)calibration for the auctions over the remaining year. Tables 3-5 
show that the achieved expected cost is approximately the same irrespective of the number of stages in all three subproblems. However, adding a number of stages reduces the variability in the solution in terms of sample standard deviation (S.D.) over all scenarios.

With 4 stages, it can be seen from table 6 that the optimization approach would have resulted in an average of $27.5 \%$ savings over the strategy of issuance used by the government. Note that the actual government debt issuance decisions are not used as input to the optimization and only the issuance mandate is used. No knowledge of the future is assumed at any stage. Thus the optimization results may be considered as out-of-sample results of the model.

\begin{tabular}{|c|c|c|c|c|c|}
\hline Q & S.D. & CaR & CVaR & S.T. & E $[I]$ \\
\hline 1 & 241.883 & $12,299.80$ & $5,050.310$ & 0.046875 & $11,762.00$ \\
2 & 182.563 & $12,175.50$ & $4,800.770$ & 0.078125 & $11,751.50$ \\
3 & 151.424 & $12,115.40$ & $4,691.210$ & 0.28125 & $11,748.00$ \\
4 & 132.354 & $12,079.50$ & $4,630.850$ & 1.4375 & $11,746.20$ \\
\hline
\end{tabular}

Table 3. Results for the short subproblem

\begin{tabular}{|c|c|c|c|c|c|}
\hline Q & S.D. & CaR & CVaR & S.T. & E $[I]$ \\
\hline 1 & $1,260.630$ & $20,067.90$ & $27,259.80$ & 0.015625 & $17,903.10$ \\
2 & 999.506 & $19,532.90$ & $26,881.80$ & 0.03125 & $17,865.80$ \\
3 & 838.865 & $19,228.10$ & $26,481.30$ & 0.046875 & $17,851.20$ \\
4 & 722.165 & $19,024.00$ & $26,316.60$ & 0.0625 & $17,845.30$ \\
\hline
\end{tabular}

Table 4. Results for the medium subproblem

\begin{tabular}{|c|c|c|c|c|c|}
\hline Q & S.D. & CaR & CVaR & S.T. & E $[I]$ \\
\hline 1 & $5,165.570$ & $71,691.90$ & $11,446.90$ & 0.3125 & $59,941.40$ \\
2 & $4,002.660$ & $69,387.50$ & $11,194.30$ & 0.046875 & $59,763.90$ \\
3 & $3,311.090$ & $68,119.10$ & $11,034.60$ & 0.0625 & $59,713.00$ \\
4 & $2,890.570$ & $67,372.10$ & $10,973.40$ & 0.203125 & $59,692.40$ \\
\hline
\end{tabular}

Table 5. Results for the long subproblem 


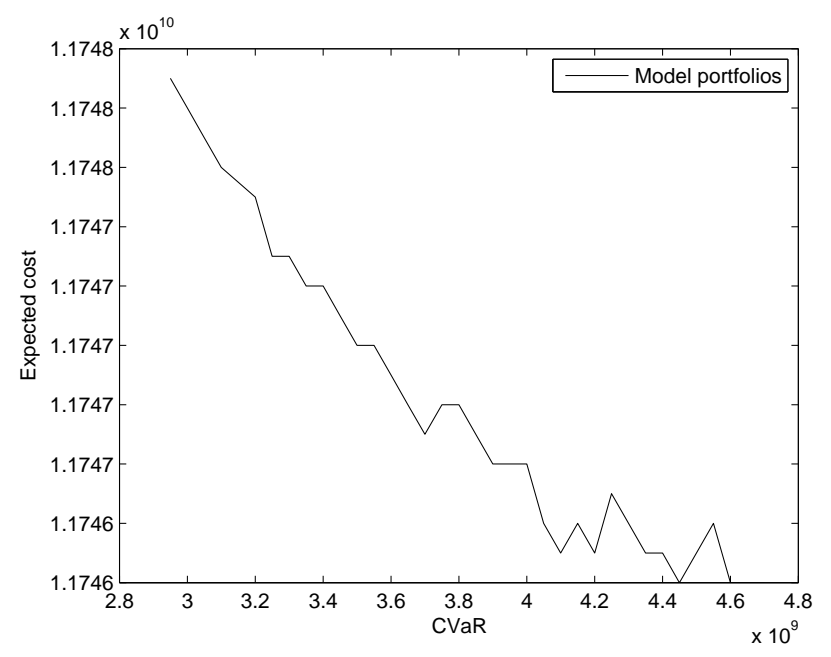

Fig. 4. Comparison of cost of issuance in sterling pounds with respect to CVaR upper bound for the short subproblem

\begin{tabular}{|l|r|r|}
\hline Subproblems & Actual cost & Model cost \\
\hline Short problem & $15,193.75$ & $11,746.20$ \\
Medium problem & $20,750.00$ & $17,845.30$ \\
Long problem & $87,207.50$ & $59,692.40$ \\
\hline Total cost & $123,151.25$ & $89,293.90$ \\
\hline
\end{tabular}

Table 6. Comparison of the cost of issuance

The above results are based on cost minimization without using a constraint on CVaR. The results for the short subproblem when an upper bound on $\mathrm{CVaR}$ is used as an active constraint are illustrated in figure 4.

As the optimization is non-convex and contains integer valued variables, the efficient frontier of expected cost vs. CVaR is highly discontinuous. The rightmost point on the graph corresponds to the CVaR unconstrained problem with $Q=4$ and the resulting $\mathrm{CVaR} \approx 4.6$ billion, as given in table 3 . By imposing a CVaR constraint and restricting CVaR to $70 \%$ of its unconstrained value results in a very modest increase in the expected cost by less than $0.1 \%$. This is partly a result of a benign economic environment; nevertheless, it shows that the CVaR constraint can be used effectively to trade-off downside cost risk against the expected cost. Similar plots are obtained for the medium and long subproblem and are omitted for brevity. 


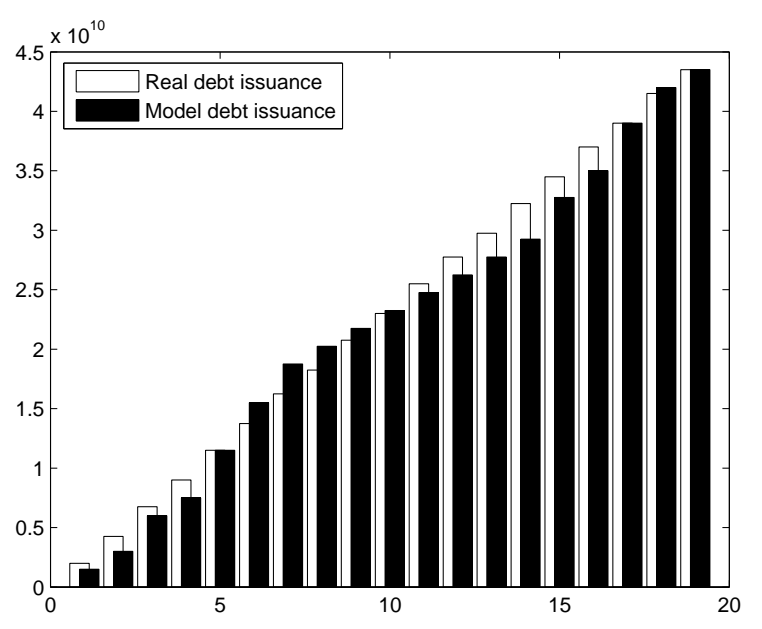

Fig. 5. Histogram of debt issuance throughout the financial year

\subsection{A posteriori analysis}

The above model proposes an optimization based approach to debt issuance. However, the issuance is often driven by exogenous factors of uncertainty, such as a change in political sentiment or macroeconomic shocks. Not all the sources of uncertainty can be adequately represented in an optimization model. From table 6 it appears that the implementation of our model would have resulted in a significant cost reduction for the UK government in the period considered. What the model does not tell is whether the implementation of the proposed cost minimization procedure leads to a maturity structure which is radically different from the one adopted in the real world. It is therefore of interest to compare our model with the actual issuance by the UK government.

Figure 5 compares the real issuance by the UK government in the auction calendar 2007-08 and the model issuance for the same calendar. At least in this instance, the model issuance does not result in large differences or strong discontinuities compared to the actual issuance. Note that the period under consideration precedes the huge increase in the financing requirement the UK government had to cover during the recent banking crisis. In general, the reliability of any model would have been stretched to the limit in the recent financial crisis.

An important exogenous factor which the government takes into account when issuing debt is the net debt to GDP ratio. The differences in the amounts issued from figure 5 are rather small. However, they have an important impact on the total cost of the issuance and can be seen in the debt to GDP ratios. The "golden rule" of the UK Treasury is to keep it below $40 \%$. However, this hasn't occurred since 2006 and an attempt to enforce it makes the problem infeasible. In figure 6, we compare the real evolution of the debt to GDP ratio compared 


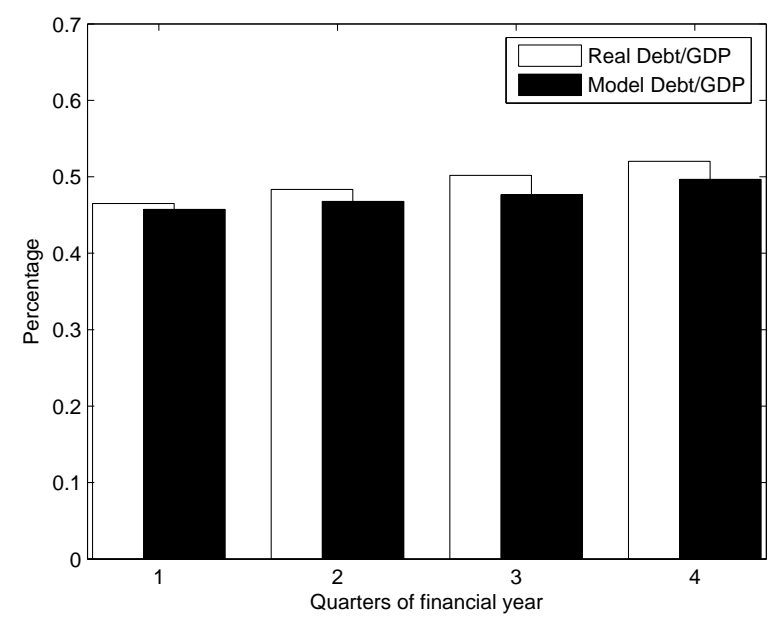

Fig. 6. Histogram of debt to GDP ratio throughout the financial year

to the model's performance. Again, we see that our simple cost minimization model doesn't have a significant adverse impact on the debt-to-GDP ratio; in fact the ratio is reduced by around $2 \%$. At least for this data-set, the $a$ posteriori analysis shows that our model performs only modestly out of line with the government strategy while achieving a significant cost reduction. This result increases the confidence in practical applicability of the model.

\section{Concluding Remarks}

In this paper we address the optimal sovereign debt issuance problem using a multi-stage, mixed integer stochastic programming model. The uncertainty in the interest rates is modeled using a re-combining lattice based on a Kalman filter calibration. The filter parameters are periodically updated to reflect the impact of issuance on the interest rate model and the optimization is repeated multiple times during the year using a receding horizon strategy. The model takes into account many of the constraints that debt managers encounter when planning the sovereign debt issuance strategy. Back-testing our model using the UK conventional government bonds shows significant reduction in the cost of debt servicing, eventually reducing the debt to GDP ratio.

Our paper extends the existing literature on debt management in several important ways:

(1) Unlike most research in sovereign debt issuance, we assume that the yields are determined by demand-side expectation (or a fair price under risk-neutral measure). Several macroeconomic variables, which can be realistically expected to affect the yields, are assumed to be read by 
the market participants who would adjust their bids accordingly. This approach avoids the need for multivariate modelling of macroeconomic variables and generation of scenarios with multiple sources of uncertainty in a stochastic optimization framework.

(2) To keep the generated scenarios arbitrage-free, we work with a latent factor based linear Gaussian model which is calibrated using a Kalman filter. The impact of debt being issued on the yield curve is implicitly accounted for by re-calibrating the yield curve model throughout the issuance period.

(3) We work in a receding horizon framework which offers a chance to recalibrate the model and re-optimize over the remaining budget year at specific dates (e.g. auction dates or periodically within the year). This leads to stochastic optimization problems of successively decreasing dimensions and also allows one to account for any modifications in the government remit during the year. This intuitively attractive approach permits a greater practical flexibility.

(4) We have also provided rigorous back-testing over a budgetary remit of an OECD country and compared the performance of our strategy with the performance of actual issuance; both in terms of costs as well as in terms of secondary effect on debt-to-GDP ratio.

There are many different directions in which this work can be extended. Perhaps the most important extension is to treat the interest rate as endogenous and model the impact of the issuance itself on the interest rate explicitly. Note that this is done only implicitly in the current model (through re-calibration). An explicit treatment of endogenous interest rates will link the more conventional macroeconomic models with our approach.

\section{Acknowledgements}

The authors are also grateful to the anonymous referees for their constructive comments which helped to improve quality of this paper.

\section{References}

Adamo, M., Amadori, A., Bernaschi, M., et al, C.C., 2004. Optimal strategies for the issuances of public debt securities. International Journal of Theoretical and Applied Finance 7, 805-822.

Babbs, S.H., Nowman, K.B., 1999. Kalman filtering of generalized vasicek term structure models. Journal of Financial and Quantitative Analysis 34, $115-130$.

Balibek, E., Köksalan, M., 2010. A multi-objective multi-period stochastic 
programming model for public debt management. European Journal of Operational Research 205, 205-217.

Barro, R., 1997. Optimal management of indexed and nominal debt. NBER Working Paper No. 6197.

Bernaschi, M., Briani, M., Papi, M., Vergni, D., 2007. Scenario-generation methods for an optimal public debt strategy. Quantitative Finance 7, 217229.

Consiglio, A., Staino, A., 2010. A stochastic programming model for the optimal issuance of government bonds. Annals of Operations Research , 1-1410.1007/s10479-010-0755-5.

Danish National Bank, 2006. Danish Government Borrowing and Debt. Technical Report. Http://www.nationalbanken.dk/dnuk/specialdocuments.nsf.

Date, P., Ponomareva, K., 2010. Linear and nonlinear filtering in finance: a review. IMA Journal of Management Mathematics In press.

Date, P., Wang, I., 2009. Linear gaussian affine term structure models with unobservable factors: Calibration and yield forecasting. European Journal of Operations Research 195, 156-166.

Diebold, F., Li, C., 2006. Forecasting the term structure of government bond yields. Journal of Econometrics 130, 337-364.

Dupačová, J., Bertocchi, M., 2001. From data to model and back to data: a bond portfolio management problem. European Journal of Operational Research 134, 261-278.

Dupačová, J., Gröwe-Kuska, N., Römisch, W., 2003. Scenario reduction in stochastic programming: an approach using probability metrics. Mathematical Programming 95, 493-511.

Golub, B., Holmer, M., McKendall, R., Pohlman, L., Zenios, S., 1995. A stochastic programming model for money management. European Journal of Operational Research 85, 282-296.

Gravelle, T., Morley, J., 2005. A Kalman filter approach to characterizing the Canadian term structure of interest rates. Applied Financial Economics 15, 691-705.

Hahm, J., Kim, J., 2003. Cost-at-risk and benchmark government debt portfolio in korea. International Economic Journal 17.

Heitsch, H., Römisch, W., 2003. Scenario reduction algorithms in stochastic programming. Computational Optimization and applications 24, 187-206.

HM Treasury, 1998. Code for fiscal stability.

Hull, J.C., White, A.D., 1994. Numerical procedures for implementing term structure models 1: single-factor models. Journal of Derivatives 2, 7-16.

James, J., Webber, N., 2000. Interest Rate Modelling. John Wiley and Sons.

Maciejowski, J., 2002. Predictive control with constraints. Prentice Hall, London, UK.

Missale, A., 1999. Public Debt Management. Oxford University Press, Oxford, UK.

Nelson, C., Siegel, A., 1987. Parsomonious modelling of yield curve. Journal of Business 60, 473-489. 
Nielsen, S., Poulsen, R., 2004. A two-factor, stochastic programming model of Danish mortgage-backed securities. Journal of Economic Dynamics \& Control 28, 1267-1289.

Risbjerg, L., Holmund, A., 2005. Advances in risk management of government debt. OECD. chapter Analytical framework for debt and risk management.

Rockafellar, R.T., Uryasev, S., 2000. Optimization of conditional value-at-risk. Journal of risk .

Rossi, G.D., 2004. Kalman filtering of consistent forward rate curves: a tool to estimate and model dynamically the term structure. Journal of Empirical Finance 11, 277-308.

The International Monetary Fund and The World Bank, 2003. Guidelines for Public Debt Management. Technical Report. IMF Publications.

Vasicek, O., 1977. An equilibrium characterization of the term structure. Journal of Financial Economics 5, 177-188.

Zenios, S., 1995. Asset/liability management under uncertainty for fixedincome securities. Annals of Operations Research 59, 77-97. 